\title{
Swelling of linear polymers in mixed swelling agents; predictability by means of solubility parameters
}

\author{
P. E. Froehling, D. M. Koenhen, A. Bantjes and C. A. Smolders \\ Laboratory for Macromolecular Chemistry, Twente University of Technology, PO Box 217. \\ Enschede, The Netherlands \\ (Received 26 March 1976; revised 19 Mav 1976)
}

\section{INTRODUCTION}

The solubility behaviour of polymers can be predicted to a reasonable extent by applying the Hildebrand relation $^{1}$ which connects the energy of mixing of two compounds to their individual energies of vaporization:

$$
\frac{\Delta E_{\mathrm{mix}}}{\Phi_{1} \Phi_{2}}=V\left(\delta_{1}-\delta_{2}\right)^{2}
$$

where $\Delta E_{\mathrm{mix}}$ is the energy of mixing; $\Phi_{1}$ and $\Phi_{2}$, the volume fractions of the components; $V$, the total volume of one mole of the mixture and $\delta_{1}, \delta_{2}$, the Hildebrand solubility parameters of both components, defined by:

$$
\begin{aligned}
& \delta=(\text { cohesive energy density })^{1 / 2} \\
& =\left(\frac{\Delta E_{\text {vap }}}{V_{m}}\right)^{1 / 2}
\end{aligned}
$$

where $\Delta E_{\text {vap }}$ is the energy of vaporization and $V_{m}$ is the molar volume. From equation (1) it is clear that a smaller difference in $\delta$ between two compounds results in a diminished energy of mixing. A decrease in $\Delta E_{\mathrm{mix}}$ gives a higher degree of swelling and eventually complete solubility may result. The applicability of equation (1) has been extended by Hansen ${ }^{2}$, who divided the solubility parameter into contributions from disperse $\left(\delta_{d}\right)$, dipole $\left(\delta_{p}\right)$ and donor/acceptor or hydrogen-bond $\left(\delta_{h}\right)$ interactions, and represented $\delta$ as:

$$
\delta^{2}=\delta_{d}^{2}+\delta_{p}^{2}+\delta_{h}^{2}
$$

which may be taken as a vector sum.

It is possible to express each of the solubility parameter components for a mixture of two solvents as:

$$
\delta_{m, i}=\Phi_{1} \delta_{1, i}+\Phi_{2} \delta_{2, i} ; \quad i=d, p, h
$$

This relation can be used only when there are no specific interactions upon mixing, since these interactions (e.g. additional donor/acceptor interactions) do not obey the geometric mean rule.

The values of the components of $\delta$ have been reported for numerous compounds, and methods to calculate them from other physico-chemical properties of solvents and polymers are progressing rapidly ${ }^{3}$.

The often encountered enhanced interaction of polymers with solvent pairs can be explained by the vector concept of the solubility parameter, of which a representation is given in Figure 1.

The affinity of a solvent mixture $X$ (on the line $\mathrm{A}-\mathrm{B}$ ) with respect to a polymer is inversely proportional to its distance to $\mathrm{P}$, the location of the polymer in the $\delta_{d}, \delta_{p}, \delta_{h}$ space. It is evident that for the given spatial configuration of $\mathrm{A}, \mathrm{B}$ and $\mathrm{P}$ there exists a point $M$ at which the energy of mixing is at a minimum and swelling should reach a maximum. The application of the solubility parameter concept to the interaction of polymers with solvent mixtures has been confined up to now to qualitative aspects of the solubility of linear polymers ${ }^{2,4}$ and the swelling behaviour of crosslinked elastomers 5 . We wish to report here on the application of the same concepts to the swelling of linear polymers by swelling agent/non-solvent mixtures.

The systems studied are shown in Table 1. Toluene, trichloroethylene and n-butyl acetate are well-known swelling agents for poly(vinyl chloride), while methanol and nitromethane are strict non-solvents ${ }^{2}$. For the combina- tion n-butylacetate/nitromethane a deviating behaviour may be expected, as these solvents show mutual interaction on account of their respective electron donating and accepting character ${ }^{6}$. The other two solvent pairs do not show specific interactions which may cause deviations.

\section{EXPERIMENTAL}

Poly(vinyl chloride), Breon S 110/10 (BP Ltd), was a very pure preparation according to i.r. spectrum and elementary analysis. From gel permeation chromatography (solvent tetrahydrofuran, stationary phase Styragel-Waters Associates) the molecular weight distribution was calculated as ${ }^{7,8} \bar{M}_{n}=$ $28000, \bar{M}_{w}=56000$ using calibration samples of PVC from Pressure Chemical Co. All solvents were analytical grade quality.

PVC films were cast from a $15 \%$ so-

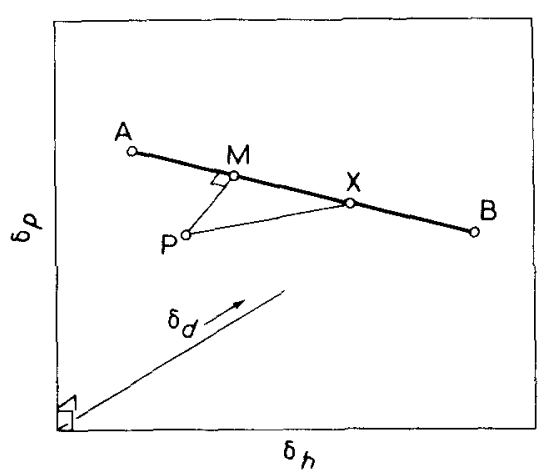

Figure 1 Spatial representation of solvent mixture/polymer interaction

Table 1 Solubility parameters of PVC and solvents. Values are taken from ref 3

\begin{tabular}{llll}
\hline Compound & $\delta_{d}$ & $\delta_{p}$ & \multicolumn{1}{c}{$\delta_{h}$} \\
\hline PVC & 9.15 & 4.9 & 1.5 \\
Toluene & 8.82 & 0.7 & 1.0 \\
Trichloroethylene & 8.78 & 1.5 & 2.6 \\
n-Butylacetate & 7.67 & 1.8 & 3.1 \\
Nitromethane & 7.70 & 9.2 & 2.5 \\
Methanol & 7.42 & 6.0 & 10.9 \\
\hline
\end{tabular}


lution in tetrahydrofuran. The dry films (about $0.5 \mathrm{~mm}$ thick) was cut into pieces of $\sim 200 \mathrm{mg}$. The pieces were immersed in the solvent mixtures, and (after rapid blotting with filter paper) weighed after 2,6 and $20 \mathrm{~h}$ immersion. Equilibrium was attained after $6 \mathrm{~h}$ at $20^{\circ} \mathrm{C}$. All experiments were carried out in triplicate.

\section{RESULTS}

The results of the swelling experiments are plotted in Figures 2-4, together with the calculated polymer/solvent distances in the $\delta$ space:

$$
\begin{aligned}
\Delta= & {\left[\left(\delta_{p, S}-\delta_{p, P}\right)^{2}+\left(\delta_{d, S}-\delta_{d, P}\right)\right.} \\
& \left.+\left(\delta_{h, S}-\delta_{h, P}\right)^{2}\right]^{1 / 2}
\end{aligned}
$$

where the subscripts $S$ and $P$ refer to solvent and polymer respectively.

Values of $\delta$ were taken from Koenhen $^{3}$. The values of the solvent mixtures were calculated according to equation (4). It is clear that for the solvent pairs toluene/methanol and trichloroethylene/nitromethane which do not show donar/acceptor interactions upon mixing, the maximum in the swelling curve coincides with the calculated composition at which the distance in $\delta$-space is minimal. The magnitude of the relative increase in swelling with relation to the pure solvents appears to be proportional to the calculated decrease in $\Delta$.

It should be pointed out that the $\delta$ values for PVC as given by Hansen ${ }^{2}$ result in a poorer agreement between the calculated and measured extreme values of $\Delta$ and degree of swelling. In the case of the donor/acceptor solvent pair

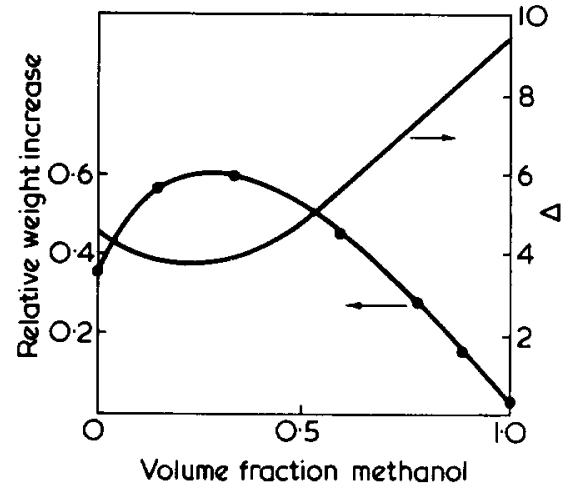

Figure 2 Calculated interaction parameter $\Delta$ and measured relative weight increase of PVC in toluene/methanol mixtures

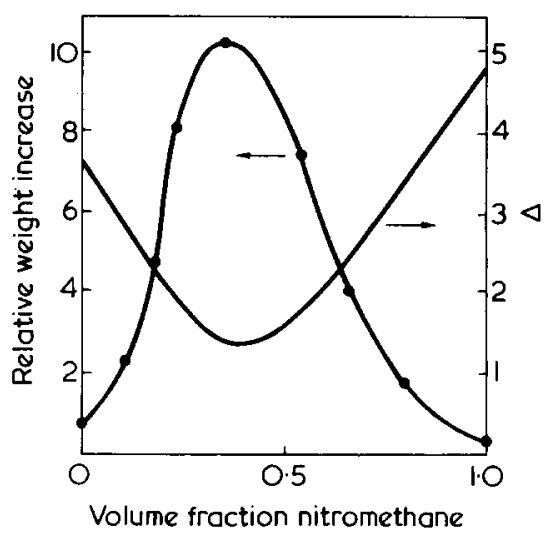

Figure 3 Calculated interaction parameter $\Delta$ and measured relative weight increase of PVC in trichloroethylene/nitromethane mixtures

n-butyl acetate/nitromethane no maximum occurs in the swelling curve, contrary to expectation.

Caution should therefore be exercised in the application of the solubility parameter concept in mixtures of solvent which may interact through donor/ acceptor forces.

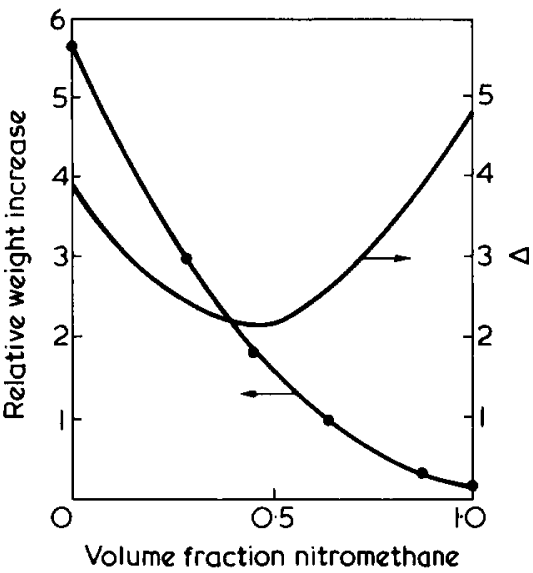

Figure 4 Calculated interaction parameter $\Delta$ and measured relative weight increase of PVC in n-butylacetate/nitromethane mixtures

\section{ACKNOWLEDGEMENT}

The g.p.c. measurements were carried out by $\mathrm{G}$. van de Ridder.

\section{REFERENCES}

1 Hildebrand, J. H. and Scott, R. L. 'The Solubility of Non-Electrolytes', Dover, New York, 1949

2 Hansen, C. M. J. Paint Technol. 1967, 39, 104

3 Koenhen, D. M. and Smolders, C. A. J. Appl. Polym. Sci. 1975, 19, 1163

4 Chen, S. A. J. Appl. Polym. Sci. 1971, 15,1247

5 Beerbower, A. and Dickey, J. R. $A S L E$ Trans. $1969,12,1$

6 McClellan, A. and Pimental, G. 'The Hydrogen Bond', Freeman, San Francisco, 1960

7 Evans, J. M. Polym. Eng. Sci. 1973, 13, 401

8 Cardenas, J. N. and O'Driscoll, K. F. J. Polym. Sci. (Polym. Lett. Edn) 1975, 13,657

\section{A method for the calculation of optimum filler content in amorphous and semi- crystalline polymers}

\section{S. C. Shea*}

Department of Polymer and Fibre Science, University of Manchester Institute of Science and Technology, Manchester, UK

(Received 22 March 1976; revised 13 Mav 1976)

\section{INTRODUCTION}

Currently more and more additives are being used in polymers, particu. larity the polyolefins. These additives include fillers, flame retardants, pig-

* Present Address: Van Leer Research Laboratories, Passfield, Hampshire, UK ments etc. Consequently, it is desirable to be able to predict the concentration of additive above which the mechanical properties significantly deteriorate. This communication details a method of calculating an optimum filler concentration using principles well known in the surface coat- ing industry. While this proposed technique will be illustrated using high den sity polyethylene, it is thought that since the equations presented here are of a general nature, they may be more widely applicable. Of the many tests available elongation at break is prob: ably the most sensitive and will be used here to compare experimental results with theory.

\section{THEORY}

Consider a polymeric material to which 